ISSN: 2349-2031

Research Article

\title{
The Effect of Trait Anxiety on Recognition of Threatening Emotional Facial Expressions: A Study among High School Students.
}

\author{
Roslinda Mustapha1, Md. Azman Shahadan² and Hazalizah Hamzah ${ }^{3}$ \\ 1,23 Department of Psychology and Counseling, \\ Universiti Pendidikan Sultan Idris, 35900 Tanjong Malim, Perak, Malaysia.
}

\begin{abstract}
Previous studies indicated that sensitivity to facial expressions of threat is related to anxiety in children, adolescents and adults. A small amount of anxiety often improves students' performance, but a high level of anxiety can cause them to perceive many daily situations as threatening and this will result in more frequent experiences of fear of what may happen, especially for the high anxiety students. This research will explore the threat perception that the secondary school students might have in relation to negative facial expression and examine the sensitivity towards anger expressions as threatening stimuli. 49 students (25 low anxiety and 24 high anxiety) age between 16 to 18 years old have been recruited to answer a set of anxiety questionnaires and they were also required to identify the facial expression to explore the threat perception by looking at images posing facial expression in 2 and 3 dimensions. These images have been transformed into 5 levels of anger using FaceGen Modeller 3.5. Results demonstrated that the high anxiety students can identify threat stimuli from faces more accurately and faster than the low anxiety students. It is suggested that angry faces may be perceived as particularly threatening amongst students and play a significant role in their emotional well being. It is hoped that this research will increase our understanding of the relationship between anxiety and threat perception and this unique visual stimulus can generate a wealth of other research in Malaysia.
\end{abstract}

Keywords: Face perception, facial expression, anxiety, angry face stimuli, threat perception.

\subsection{Introduction}

Face perception has long been an interesting field of study. A lot of previous research has found that anxiety significantly correlated with threat perception (Gutierrez-Garcia \& Calvo, 2016; Schofield, Johnson, Inhoff \& Coles, 2012). Beside faces, other ambiguous stimuli such as body gesture, stories, voices, words, animals or threat related situation were also used (LoBue \& Perez-Edgar, 2015; Koizumi, Tanaka, Imai, Hiramatsu, Hiramoto, Sato and Gelder, 2011; Bar-Haim et al., 2007).

Individual with anxiety trait are more sensitive to a variety of threatening stimuli when compared to non-anxious individuals, and they tend to interpret others' emotional state in a negative manner (Richards et al, 2002) which include the mixed emotion such as fear and surprise and neutral faces that are ambiguous in their thinking (Mohlman et al. 2007; Yoon and Zinbarg 2008). While many studies examined the bias of anxious individuals in recognizing facial expression, few studies have examined the sensitivity in detecting threatening facial expressions. The ability and the sensitivity towards recognizing facial emotions is an important component in our nonverbal communication system. It is also a crucial social skill needed for every human being from infancy stage to adult stage to successfully adapt and manipulate the environment. Unable to correctly interpret the emotional facial expression will Adolescent stage is especially important as this stage of development has long been recognized as a period of heightened risk-taking, a stage that need oversight from adults. What students had experienced daily at schools might be perceived as threatening especially by the anxious students. They probably even interpreted words and neutral facial expression around them in a negative way or as a sign of threat or danger. According to Wieser \& Moscovitch (2015), neutral faces may produce the feeling of anxiety for socially anxious individuals due to their ambiguous nature. There are many different kinds of anxiety that can be identified among schools' student as well as the causes of anxious feeling experienced by them. Anxiety can be caused by different factors such as biological, cognitive and psychological factors that are intertwined in a complicated ways such as examination, test, pressure of school work, peers, family problems or parents divorcing can all be difficult events for students and can be a sources of anxiety for them (Nadeem, Ali, Maqbool, \& Zaidi, 2012).

However, to our knowledge, what does not seem to have been investigated is the effect of threat perception on anxious students. Therefore, this study aims to gain a better picture and understanding of students' anxiety and how it's effect their perceptions of negative facial expression. The aims of the study can be stated specifically into four objectives:

1. To investigate if there is a significant interaction between anxiety, in a way that students with high anxiety will readily recognize angry expression accurately than students with low anxiety.

2. To investigate whether there is a significant interaction between groups of anxiety and threat perception across five morphs levels of anger expressions. 
3. To investigate if there is a significant interaction between anxiety, in a way that students with high anxiety will detect angry expression faster than students with low anxiety.

4. To investigate whether there is a significant interaction between groups of anxiety and response time across five morphs levels of anger expressions.

\subsection{Methodology}

The study has been conducted by using experimental method. The STAI-trait anxiety questionnaire was used for pre-screen to classify the high and low anxiety students. The data were collected from the face perception tasks to examine whether there are differences between the high and low anxiety students in perceiving the threat from face stimuli.

Participants were selected among students from three different secondary schools in Selangor area. The schools were selected because of the availability of form 4 and form 6 students that is consistent with the study's model background that targeted adolescent participants age 15 to 19 years old. 137 (53 male and 84 female) students age predominantly 16 and 18 years old were recruited in order to conform to the restrictions imposed by the Ministry of Education regarding involvement of exam year students (which inadvertently excluded Form Three and Five students). Only participants with STAI-Trai anxiety scores over 75 and under 25 percentile were selected. The final samples were composed by 25 high anxiety students and 24 low anxiety students. We were helped by school teachers and counselor in providing samples, preparing the room for the tests and gathering other information needed for this study.

All participants completed the demographic questionnaires and State Trait Anxiety Inventory (STAI-state \& trait; Spielberger, 1983). The Instrument consists of 40-item self-report questionnaire with two subscales namely as state anxiety and trait anxiety. This questionnaire was used to measure participants' social anxiety level. STAI is a self-report measures that can assess how respondents "feel right now, at this moment" (y1) and how respondents "generally feel" (y2). According to Spielberger (1989), the STAI can also identify the high and low anxiety and has appeared in over 3,000 studies and has been translated into 30 languages (Gros, Antony, Simms, \& McCabe, 2007).

\subsection{Selection of angry facial expression}

There are numbers of study have found the advantage of angry or threatening face over other facial expression such as happy or neutral faces (LoBue, 2009; Harvey, Thrasher, LoBue, \& Matthewa 2014) or sad and fearful faces (Calvo, Avero, and Lundqvist, 2006). Those studies had covered all group of ages, from infancy, children, adolescent and adults. Impressively, even infants as young as 9 months can detect angry faces more quickly than happy, neutral and sad faces (LoBue, 2009, LoBue, 2010). Many researchers have suggested that the rapid detection of threatening faces from angry expression is because of its threatening message produced by the angry face stimuli (LoBue et al., 2013). Even more, the anxious individuals have interpreted the neutral facial cues as conveying anger emotion regardless the threat condition (speech condition), while the non-anxious individuals have interpreted the neutral faces in a negative manner only when they were in the threat condition (Yoon \& Zinbarg, 2008).

\subsection{Generating facial stimuli}

The material for the face task was actually duplicated from study by Mustapha, Shahadan, Abdul Wahab Khan, Stephan \& Archer (2017). The face stimuli were 6 faces consist of 3 male faces and 3 females' Malay faces showing neutral expressions. Those photos of real life face showing neutral expressions of emotion were converted into virtual avatars using the "photo fit" application in the FaceGen. FaceGen is a 3D facegenerating software (http://facegen.com). We used this FaceGenModeller software, version 3.1 because it can generate a large number of stimuli easily and the users are allowed to randomize, normalize, generate emotional facial expression systematically and adjust certain characteristic such as race, gender, age, eye gaze and head position (Oosterhof \& Todorov, 2008). The models are of medium age $(25+$ years old $)$, bare of hair on the external features. We then generated 4 levels of anger from each of the neutral faces using an automated procedure in FaceGen. The morphing degree was adjusted into anger expression and the intensity of emotion was added linearly started with a neutral face at $0 \%$ of anger to $25 \%, 50 \%$, $75 \%$ and $100 \%$ of anger resulting in 4 morphed images from each neutral face. Thus, there were 6 sets of facial images that had been generated into 30 emotionally angry faces of male and female stimuli (6 faces x 5 level of anger emotion strength). The example of one set of the generated faces is presented in figure 1. The face stimuli were then presented randomly in the PsychoPy program for the experiment. We presented the facial images for $2000 \mathrm{~ms}$. The facial images were 400 X 400 pixel size and the images were presented in the middle of the screen with a black background.

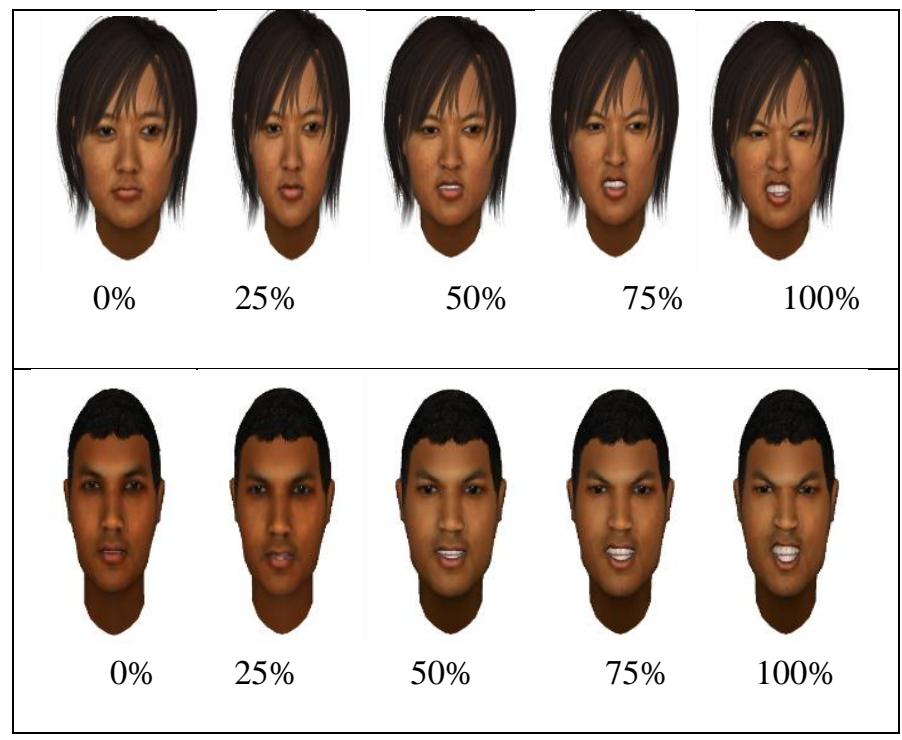

Figure 1: Example of unfamiliar facial images as stimuli that have been generated into five levels of anger $(0 \%, 25 \%, 50 \%, 75 \%$ and $100 \%)$. Adopted from Mustapha et al. (2017)

\subsection{Procedures}


The experiment was run using a psychoPy program and was set up on the laptop as it will be easier for us to conduct the experiment in a school setting. The face stimuli were presented randomly and the measure of the answer is based on the proportions of "yes" responses. The study was approved by the Ethics Committee of University of Nottingham Malaysia Campus and granted permission by the Ministry of Education, Selangor State Department of Education and the respective school principal.

The nature and purpose of the study were explained to participants briefly and how the experiment will be conducted. They were then asked to complete the set of questionnaires consisting of: section 1 = Demographic Questionnaire, section 2 = State-Trait Anxiety Inventory (STAI); and doing the experiment of face perception. Approximately 20 to 30 minutes is needed by the participants to complete the questionnaires. The questionnaires were made available in Malay Language because many of the students are not good in English language.

The facial stimuli task was administered upon completing the questionnaires. Participants were instructed about what they will see on the screen and what they have to do. Participants were presented with images of 30 faces showing facial expressions, one facial image at a time. They were presented with a fixation point $(+)$ for $1000 \mathrm{~ms}$ at the center of the screen, followed by facial image for $2000 \mathrm{~ms}$. After that, the participants were asked if the facial image shows angry emotion or not angry. The task was self-paced where the question remained on the screen until the participant's response. Participants made their responses by pressing the " $Z$ " key on the keyboard which was labeled "yes" if the answer is angry and "M" key which was labeled "No" if the answer is not angry. The order of the trials was randomized for each participant. Participants were instructed to press the "yes" key or the "no" key as quickly as possible while still being accurate. RTs were recorded automatically by the computer. The average time needed to complete the task is less than 20 minutes. All the instruction given to the participants were in Malay language.

\subsection{Result}

The Analysis was conducted using the Statistical Package for Social Sciences version 21 (SPSSv21). Initial analyses examining the relations between demographic variables and student' self-reported anxiety included independent t-test for categorical variables. For the face experiment, we used a standard visual search procedure in which the accuracy in identifying emotional expressions had been measured based on the proportion of 'yes' responses when the participant chooses the target emotions per level of morphs. The mean of reaction time had been calculated as a function of sensitivity toward threat stimuli. In order to examine whether there is differences between the mean scores of identifying angry expression from five levels of anger expressions, a one-way within-group ANOVA was computed (Field, 2009). Finally, repeated measure analysis of variance (ANOVA) with group (high vs low anxiety) by emotion valence (five morph levels) have been conducted to test the predictions about the ability of students in recognizing angry facial expression as well as its response time. The dependent variables are the proportion of the correct answers and response time.

\subsection{Preliminary analysis}

The participants were divided into high and low anxiety groups. For the prescreen session, 137 (53 male and 84 female) students age between 16 to 18 years old with a mean age of 17.4 ( $\mathrm{SD}=1.43$ ) were recruited. Only participant s with STAItrait anxiety scores over the 75 and under 25 percentile were selected. The final sample was composed by 24 participant (10 male and 14 female and 25 participants $(7$ male and 18 female). The two groups differ significantly on STAI-trait scores $\mathrm{t}(47)=-21.2, \mathrm{P}<0.01$, STAI-state score $\mathrm{t}(47)=-3.46$, $\mathrm{P}<0.01$. However, they did not differ significantly in age $\mathrm{t}(47)=6.71, \mathrm{P}>0.01)$ respectively (refer to table 1$)$.

Table 1: Mean scores and standard deviation of STAITrait and STAI-State of anxiety.

\begin{tabular}{lllll}
\hline & \multicolumn{3}{l}{ High Anxiety Student } & $\begin{array}{l}\text { Low } \\
\text { students }\end{array}$ \\
& Mean & SD & Mean & SD \\
\hline STAI-trait & 57.2 & 5.3 & 28.1 & 4.2 \\
STAI-state & 48.7 & 8.3 & 39.3 & 10.5 \\
Age & 16.4 & 1.04 & 18.4 & 1.01 \\
\hline
\end{tabular}

Prior to the main analysis we have first examine the effect of morph levels of the face stimuli to determine whether it can be a potential tool to facilitate students in making in identifying threatening stimuli. Therefore, a one-way within-group ANOVA was computed. The result showed that there was a statistically significant differences between the score of identifying angry faces across five morph levels of angry expression, Wilks' Lambda=.088, F(4, 46)= 262.07, p<.001.

Since we have found a significant result for overall differences, we then computed additional paired samples t-tests to make post hoc comparisons between each levels. A first paired samples t-test indicated that there was a significant difference in the scores for neutral $(\mathrm{M}=.89, \mathrm{SD}=1.37)$ and $25 \%(\mathrm{M}=1.45$, $\mathrm{SD}=1.36)$ morph levels; $\mathrm{t}(49)=-4.210, \mathrm{p}<.001$. A second paired samples t-test indicated that there was a significant difference in the scores for $25 \%$ and $50 \%(\mathrm{M}=2.67, \mathrm{SD}=1.35)$ morph levels; $\mathrm{t}(49)=-7.212, \mathrm{p}<.001$. A third paired samples $\mathrm{t}-$ test indicated that there was no significant difference in the scores for $50 \%$ and $75 \%(\mathrm{M}=4.78, \mathrm{SD}=1.45)$ morph levels; $\mathrm{t}(49)=-13.050, \mathrm{p}>.001$ and finally, a forth Paired Samples TTest indicated that there was a significant difference in the scores for $75 \%$ and $100 \%(M=6.23, \mathrm{SD}=1.39)$ morph levels; $\mathrm{t}(49)=-8.764, \mathrm{p}<.001$. Therefore, this stimuli has showing a strong effect of all morphs level an applicable to predict the differences in perceived threat.

\subsection{Main Analysis}

\subsubsection{Emotional Recognition Task}

There was a statistically significant difference for STAI-trait anxiety score between high $(\mathrm{M}=17.52, \mathrm{SD}=3.82)$ and low 


\section{Roslinda Mustapha et. al / The Effect of Trait Anxiety on Recognition of Threatening Emotional Facial Expressions: A}

Study among High School Students.

$(\mathrm{M}=15.21, \mathrm{SD}=3.71)$ in terms of total angry scores, $\mathrm{t}(47)=$ 2.15, $\mathrm{p}=.037$ (see figure 2a). This means that in general, students with high anxiety can identify the anger expression of the facial image more accurately than the students with low anxiety. Further analysis was conducted in order to test whether there are also differences between the anxious groups in perceiving threat perception across five morph levels. Repeated measure ANOVA of accuracy of facial expression identification showed no significant main effect of group $F$ $(1,47)=3.71, p>0.05$. However, there is a significant effect of morph levels $\mathrm{F}(4,3.21)=158.4, \mathrm{p}<0.001$; and a significant interaction of anxiety group x morph levels, $F(4,3.21)=2.95$, $\mathrm{p}<0.05$. There were no other interactions of variable as a function of anger expression identification. According to several pairwise comparison, there are significant differences between all level of facial morphs with $\mathrm{p}<0.05$. Specifically between neutral and $25 \%$ of morph levels $(\mathrm{p}<0.001)$, between $25 \%$ and $50 \%$ of morph levels $(\mathrm{p}<0.001)$, between $50 \%$ and $75 \%$ of morph levels $(\mathrm{p}<0.001)$ and between $75 \%$ and $100 \%$ of morph levels $(\mathrm{p}<0.001)$. The interaction effect suggested that the high anxiety students showed more accuracy in detection of angry face when presented with ambiguous stimuli, specifically towards $50 \%$ and $75 \%$ of morphs levels.

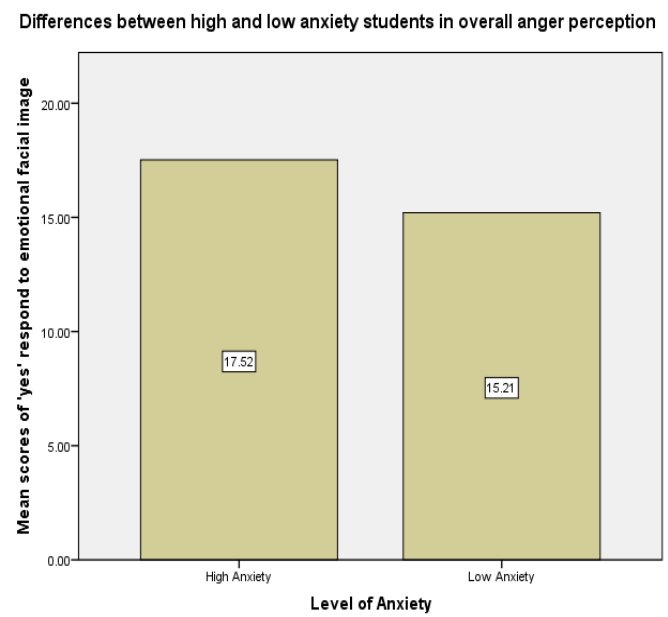

Figure 2a: The differences in accuracy to detect anger facial expression between students with high and low anxiety

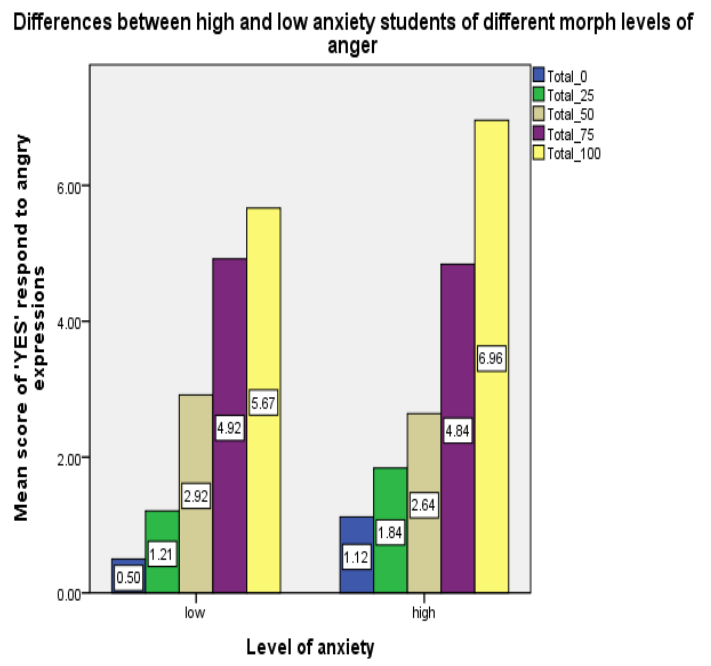

Figure 2b: The differences in accuracy to detect anger facial expression between students with high and low anxiety across five morph levels

\subsubsection{Respond Time}

There was a statistically significant difference between high $(\mathrm{M}=19.5, \mathrm{SD}=8.48)$ and low $(\mathrm{M}=39.31, \mathrm{SD}=17.91)$ stai-trait anxiety score in terms of total time needed to identify anger expression, $\mathrm{t}(47)=4.96, \mathrm{p}=.000$. it was shown that students with low anxiety spent more time to recognize the anger expression from the images as compared to the high anxiety students (figure 3a).

Further analysis was conducted to examine whether there is also differences in response time between the two anxious groups across five levels. Repeated measure analysis of ANOVA have showed no significant main effect of group $\mathrm{F}$ $(1,47)=.003, p>0.001$. and no significant interaction of anxiety group $x$ morph levels, $F(4,2.71)=.481, p>0.05$. However, there is a significant main effect of morph levels $F(4,2.71)$ $=4.005, \mathrm{p}<0.005$ (see figure $3 \mathrm{~b}$ ). The main effect of morph levels showed that there were significant differences between the five morph levels. According to several pairwise comparisons, there is significant differences between neutral and $50 \%$ of morph level ( $\mathrm{p}<.05)$, between neutral and $100 \%$ of morph level $(\mathrm{p}<0.05)$. In other words, There are no other significant differences between other morph levels.

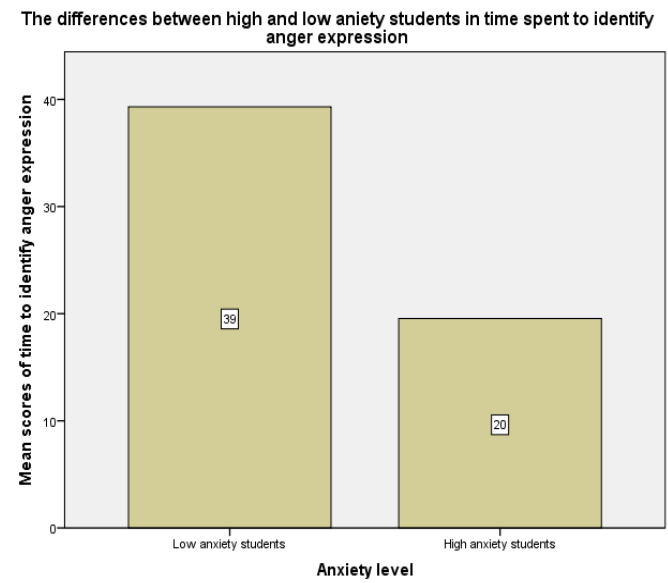

Figure 3a: The differences in reaction time to detect anger facial expression between students with high and low anxiety

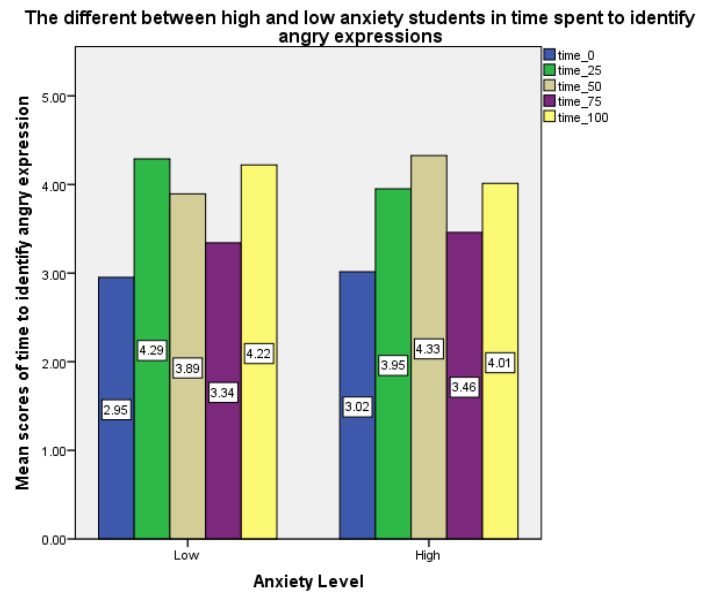

Figure 3b: The differences in reaction time to detect anger facial expression between students with high and low anxiety across five morph levels 

Study among High School Students.

\section{Discusion}

\subsection{The accuracy of detecting angry expressions}

The results from the analysis of accuracy in detection of angry emotions appeared to be consistent with the hypothesis. Based on the total scores of accuracy, students with high anxiety can identify anger expressions of the facial images more accurately than the students with low anxiety (hypothesis 1). Even, when participants were displayed with different levels of anger intensity (from neutral to $100 \%$ of anger expression), the results also indicated a significant differences between the anxious groups in regards with accuracy of the detecting the threat related expressions. The anxious participants had also been able to identify angry expression accurately at a $50 \%$ of morph level which was expected to act as ambiguous stimuli. The results showed that individuals with high social anxiety overestimate perceived social danger even from subtle facial cues.

This finding seems compatible with the intentional bias towards threatening faces in anxious participants found in several other studies. For example, Cisles \& Koster (2010) in their study had found that anxious individuals are more likely to allow threat-related information (in the form of words or faces) to unduly control their thoughts and actions. A study by Mohlman, Carmin \& Price (2006), also found that participants with generalized social anxiety disorder (GSAD) sorted angry cards with greater accuracy as compared to the non-anxious control samples. There was also evidence that the GSADs tend to misclassified neutral cards as angry. Similarly, Schofield et. al. (2012), also found that social anxiety was associated with difficulty disengaging attention from threatening facial expressions as they combined a dot-probe task that required participant to disengage their attention by tracking the eyemovement. The result indicated that this response strategy may be related to adaptive strategy for preparing for potential threat before accounting it (Mobbs et. al. 2009). Meaning that, the high anxiety students have a tendency to be cautious about potential threat in advance regardless of the actual threat evidence in the stimuli.

According to Koizumi, Tanaka, Imai et. al. (2009), individuals with high trait anxiety were found to have a tendency to interpret others' emotional states negatively when the participants were presented with dynamic faces and voices in a synchrony pattern. The anxious individuals were also showed bias in their recognition of the face and voice stimuli if the images displayed in parallel, but not if they were presented in isolation. This recognition bias in high anxiety students is not generalized to recognition of emotional faces regarding to neural expression, but it is specific to angry. As regards to the high anxiety individuals, Pollak et al. (2005) suggested that individuals who had been exposed to the multiple life aversively events require more intense facial expression of anger due to the low intensity of angry stimuli because they are normally accustomed to more severe levels of anger expression. While the above studies were all conducted in a non-realistic situation, there were at least three studies examining the effect of anxiety on perception under a more naturally occurring condition. MacLeod, Mathews, \& Tata, (1986), MacLeod and Mathews (1988) and Mogg, Bradley, and Hallowell (1994) had examined the attentional bias under condition of exam stress. The high anxiety students were tested one week before the exam and low anxiety students 12 week before the major exam. The result showed that the high anxiety students attended to the exam related words presented to them, while the low anxiety students avoided such words. In regards to developmental aspect, the result had strongly supported by other study from Lobue et al. (2014) whom had found that both children and adults detect angry adult faces as a threatening emotions faster than sad and happy faces regardless of whether they are posed by adults' or children's face stimuli.

However, several research have revealed contradict finding such as a studies by Schofield, Coles, \&Gibb (2007) has explained that anxiety doesn't have any impact on the detection of negative facial expressions and in a study by Philippot \& Douilliez (2005) they have found that anxiety is also not biased in evaluating facial expressions more negatively. Despite of no differences between non-anxious participants and socially anxious participants found by the above researchers, the socially anxious participants systematically used additional visual information from eyes, nose and mouth regions to reach the same performance level as non-anxious participants (Langner, Becker, and Rinck, 2009). In addition, Richards, Hadwin, Benson et. al. (2011) found that the high level of anxiety are associated with the increased processing capacity to detect multiple angry faces, even with the absence of threat, the anxious individuals tend to focus their attention in order to facilitate the detection of threatening stimuli in various conditions or locations to minimize the potential danger. The study also highlight that the high anxiety students have no deficit for discriminating neutral and angry expressions based on the correct response. Moreover, according to Quigley et al. (2012), the high anxious individuals might readily appraise the possible threat as the situation itself was not objectively threatening. Thus, the accuracy of detecting threat from angry expression may be more evident if the threat value of the stimuli is more difficult to appraise such as unpredictable or ambiguous stimuli.

\subsection{The response time in detecting angry expressions}

The hypothesis predicted that high anxiety students will detect anger facial expression faster than the low anxiety students. The result had also confirmed that the students with low anxiety spent more time to recognize the anger expression from the facial images as compared to the high anxiety students (hypothesis 3). A large number of previous studies reported that the unpleasant stimuli, including angry facial expression have indicated that anxious individuals took less time to identify threatening stimuli. For example, a study by Langner et al. (2015) in which the high anxious participants were faster overall in recognizing expressions across lower frequency band. However, according to Heimberg, 1995 in Langner et al, 2015, the participants have speed advantage in their performance, partly because they may become so eager to 
perform well in the face experiment. This phenomena is known as floor effect, in which socially anxious individuals seem to be more susceptible to the demand characteristic of experiments. Whereas, based on four experiments conducted by Fox, Lester, Russo, Bowless, Pichler \& Dutton, 2000, presented kind of fairly clear evidence that angry faces detected more efficiently and rapidly compared to happy and neutral faces regardless of the used of schematic faces as stimuli through visual search experimental paradigm. The research also found that the detection of angry faces had speed up when they appear among three to seven neutral or happy distractors. The results also supported by Leber, Heidenreich, Stangier \& Hofmann, 2009, confirming that individuals with social anxiety were faster than non anxious individuals when they were confronted with social-evaluative threat. However, both groups did not differ in response time to detect angry faces when they were under the non-threat conditions.

When the data analyzed further across the morphs levels, it seems that there is no significant effect of groups and no interaction between the anxious groups and the morphs levels. However, the significant effect was found in morph level, specifically between the neutral faces with $50 \%$ and between neutral faces and $100 \%$ of morph level. The data showed that the low anxiety students were faster in detecting angry expression at $50 \%$ of morph levels, while the high anxiety students were faster in detecting angry expression at $100 \%$ of morph level. Interestingly to learn that at $50 \%$ of morph level which is considered as ambiguous facial expressions had been perceived rapidly as threatening among the low anxiety students. It could be explained based on several studies that have already shown that the more aversive or negatively adults rate threatening stimuli, the more quickly they detect them (Beaver et al., 2005; Lundqvist \& Öhman, 2005). Looking at the pattern of responding across different facial expressions, LoBue et al (2014) found that both adults and children detected angry faces the most quickly compared to happy and sad faces. Other explanation might due to the unnatural face stimuli generated through the process of morphing into different levels of angry expressions. It may produce intermediate facial construction that would not naturally present to the participants. Thus, there is no evidence that can be referred distinguishing the effectiveness of morphed vs. natural transitions of facial expressions. This explanation needs further investigation. Several limitations of the present study should be highlighted. First, the study was done with pre-selected students who scored not as high as expected on the STAI trait and STAI state. Naturally, the missing of selective effect on threatening facial expressions could be due to this fact. It could be suggested for further research with more socially anxious students in the future. Second, another limitation is that the female participant in this research is outnumbers the male participants due to the over-representation of female students. This of course hampers the generalizability of the results. Altogether, the present results showed that anxious students' exhibits greater perceptual sensitivity and response times to detect angry expression as threat related stimuli. This pattern of results broadly construed high anxiety students are having a tendency to interpret stimuli as threatening.

\subsection{Conclusion}

These study are crucial to our understanding about how facial expression could also be a source of threat for the anxious students. Finding of threat perception in Malaysia context allows us to reconstruct our own notions about how to interpret the data in terms of culturally driven ideas about the way adolescent might perceive people around them. This area of research would benefit from a more detailed look at the research design and the development of facial stimuli by using different face generated software that can produce better facial images for the facial emotional stimuli. This study has also provided some insights to future study. The finding should be taken as a guideline for future study rather than as definite answers to the research questions. Further study need to be conducted in order to verify the present findings by taking into accounts the recommendations for future study which has been suggested.

\section{Acknowledgements}

This work was funded by University Grant (Grant code: 20170269-107-01) from the Sultan Idris Education University. We are grateful to Research Management \& Innovation Centre (RMIC) for all the assistance given in completing this research.

\section{References}

1. Beaver, J. D., Mogg, K., \& Bradley, B. P. (2005). Emotional conditioning to masked stimuli and modulation of visuospatial attention. Emotion, 5, 67-79.

2. Calvo, M., Avero, P., \& Lundqvist, D. (2006). Facilitated detection of angry faces: Initial orientation and processing efficiency. Cognition and Emotion, 20, 785?811.

3. Fernandes Jr., O., Portugal, L.C.L., Alves, R.C.S., Campagnoli, R.R., Mocaiber, I., David, I.P.A., Erthal, F.C.S., Volchan, E., Oliveira, L. and Pereira, M. (2013). How you perceive threat determines your behavior. Frontiers in Human Neuroscience. Vol. 7, article 632.

4. Field, A. (2009). Discovering Statistics Using SPSS. (Third Edition). London: SAGE Publications Ltd.

5. Fox, E., Lester, V., Russo, R., Bowless, R.J., Pichler, A., \& Dutton, K. (2000). Facial Expressions of Emotions: Are Angry Faces Detected More Efficiently?. Cognition and Emotion, 14 (1), 61-92.

6. Gros, D. F., Antony, M. M., Simms, L. J., \& McCabe, R. E. (2007). Psychometric properties of the State-Trait inventory for cogntiive and somatic anxiety (STICSA): Comparison to the State-Trait anxiety inventory(STAI). Psychological Assessment, 19(4), 369-381. doi:10.1037 /1040-3590.19.4.369

7. Gutierrez-Garcia, A and Calvo, M. (2016). Social anxiety and trustworthiness judgments of dynamic facial expressions of emotion. Journal of Behavior Therapy and Experimental Psychiatry. Vol. 52, pp. 119-127.

8. Koizumi, A., Tanaka, A. Imai, H., Hiramatsu, S., Hiramoto, E., Sato, T. \& Gelder, B.D. (2011). The effect of anxiety on the interpretation of emotion in the face- 
voice pairs. Exp. Brain Res. DOI 10.1007/s00221-0112668-1

9. Langner, O., Becker, E.S.,\&Rinck, M. (2009). Social Anxiety and Anger Identification: Bubbles Reveal Differencial Use of Facial Information with Low Spatial Frequencies. Psychological Science,20(6), 666-670. Doi: PSCI2357 [pii]

10. Langner, O., Becker, E.S., Rinck, M. \& Knippenberg, A. V. (2015). Social Anxious individuals discriminate better between angri and neutral faces, particularly when using low spatial frequency information. Jounal of Behavior Therapy and Experimental Psychiatry,46(6), 4449.

11. Leber, S., Heidenreich, T., Stangier, U., \& Hofmann, S.G. (2009). Processing of Facial Affect Under Social Threat in Socially Anxious Adults: Mood Matters. Depression and Anxiety. Vol. 26, 196-206.

12. LoBue, V. (2009). More than just a face in the crowd: Detection of emotional facial expressions in young children and adults. Developmental Science, 12,305-313.

13. LoBue V. What are we so afraid of? How early attention shapes our most common fears. Child Development Perspectives. 2013; 7:38-42.

14. LoBue, V. \& Perez-Edgar, K. (2014). Sensitivity to social and non-social threat in temperamentally shy children atrisk for anxiety

15. LoBue, V., Matthews, K., Harvey, T. \& Thrasher, C. ( 2014). Pick on someone your own size: The detection of threatening facial expressions posed by both child and adult models. Journal of Experimental Child Psychology. 118. 134-142.

16. Lundqvist, D., \& Öhman, A. (2005). Emotion regulates attention: The relation between facial configurations, facial emotion, and visual attention. Visual Cognition, 12, $51-84$

17. MacLeod, C., Mathews, A., \& Tata, P. (1986). Attentional bias in emotional disorders. Journal of Abnormal Psychology, 95,15-20

18. MacLeod, C., \& Mathews, A. (1988). Anxiety and the allocation of attention to threat. Quarterly Journal of Experimental Psychology: Human Experimental Psychology, 40, 653-670.

19. Mobbs, D., Marchant, J.L., Hassabis, D., Seymour, B., Tan, G., Gray, M. (2009). From threat to fear: the neural organization of defensive fear system in human. Journal of Neuroscience, 29(39), 12236-12243.

20. Mogg, K., Bradley, B. P., \& Hallowell, N. (1994). Attentional bias to threat: Roles of trait anxiety, stressful events, and awareness. Quarterly Journal of Experimental Psychology, 47A, 841-864.

21. Mohlman, J., Carmin, C.N. \& Price, R.B. (2007).Jumping to interpretations: Social anxiety disorder and the identification of emotional facial expressions. BEHAVIOUR RESEARCH AND THERAPY Volume:45, Issue: 3 Pages: 591-599

22. Nadeem, M., Ali, A., Maqbool, S. \& Zaidi, S.U. (2012).
Impact of anxiety on the academic achievement of students having different mental abilities at University level in Bahawalpur (Southern Punjab) Pakistan. International online journal of educational sciences, 4(3), 519-528. ISSN: 1309-2707

23. Oosterhof, N. N., \&Todorov, A. (2008). The functional basis of face evaluation.Proceedings of the National Academy of Sciences of the United States of America, 105(32), 11087-92. doi:10.1073/pnas.0805664105

24. Philippot, P., \& Douilliez, C.(2005). Social phobics do not misinterpret facial expression of emotion. Behavior Research and Therapy, 43, 639-652.

25. Pollak, S. D., Cicchetti, D., Homung, K., \& Reed, A. (2005). Recognizing emotion in faces: Developmental effects of child abuse and neglect. Developmental Psychology, 36, 679-688.

26. Quigley, L., Nelson, A. L., Carriere, J., Smilek, D.,\& Purdon, C. (2012). The effects of trait and state anxiety on attention to emotional images: An eye-tracking study. Cognition \& Emotion, 26(November 2013), 1-22. http://doi.org/10.1080/02699931. 2012 .662892

27. Richard, H.J, Hadwin, J.A., Benson, V., Wenger, M.J. \& Donnelly, N. (2011). The influence of anxiety on processing capacity for threat detection. Psychon Bull Rev, 18:883-889. DOI 10.3758/s13423-011-0124-7

28. Mustapha, R., Shahadan, M.A., Abdul Wahab Khan, R.K., Stephan, I \& Archer, M. (2017). Students' anxiety: An examination of the perception of facial threat and perceived paternal and maternal parenting style. Journal of Research, Policy \& Practice of Teachers \& Teacher Education. Vol. 7, No. 2, December 2017, 19-32

29. Schofield, C.A., Johnson, A.L., Inhoff, A.W. \& Coles, M.E. (2012) Social anxiety and difficulty disengaging threat: Evidence from eye-tracking. Cognition and Emotion: vol. 26 (2), pp.300-311.

30. Schofield, C.A., Coles, M.E., \& Gibb, B.E. (2007). Social anxiety and interpretation biases for facial displays of emotion: Emotion detection and ratings of social cost. Behavior Research and Therapy, 45, 2950-2963

31. Spielberger, C. D. (1983). Manual for the State-Trait Anxiety Inventory (STAI). PaloAlto, CA: Consulting Psychologists Press.

32. Weiser, M. J. and Moscovitch, D.A. (2015). The Effect of Affective Context on Visuocortical Processing of Neutral Faces in Social Anxiety. Front. Psychol. vol. 6, pp. 1824.

33. Yoon, K.L. \&Zinbarg, R.E. (2008). Interpreting Neutral Faces as Threatening is a Default Mode for Socially Anxious Individuals. Journal of Abnormal Psychology. Vol. 117, No. 3, 680-685 0021-843X/08/\$12.00 DOI: 10.1037/0021-843X.117.3.68026 (2), pp.300-311. 